\title{
Aprendizaje-servicio y desarrollo cívico-social en titulaciones universitarias del ámbito educativo: preparando al alumnado para la práctica de una educación inclusiva*
}

\author{
Ígor Mella-Núñez \\ Anaïs Quiroga-Carrillo \\ Julia Crespo Comesaña \\ Universidad de Santiago de Compostela. España. \\ igor.mella@usc.es \\ anais.quiroga.carrillo@usc.es \\ juliamaria.crespo@usc.es
}

Recibido: 9/7/2020

Aceptado: 10/2/2021

Publicado: 5/7/2021

\section{Resumen}

En esta investigación se analiza el potencial que tiene la metodología del aprendizajeservicio (ApS) para desarrollar competencias de carácter cívico-social vinculadas con el ejercicio de una práctica inclusiva en futuros profesionales de la educación. Siguiendo un diseño cuasiexperimental (pretest-postest), se compara a los alumnos de titulaciones de Ciencias de la Educación de la Universidad de Santiago de Compostela (USC) participantes en experiencias de $\mathrm{ApS}$ con aquellos que siguen una vía formativa más convencional. La muestra está conformada por un total de 336 sujetos (188 en el grupo experimental y 148 en el control). Específicamente, se analizan las dimensiones de liderazgo, conducta prosocial, competencia intercultural y trabajo en equipo. Los resultados demuestran que quienes participan en $\mathrm{ApS}$ terminan superando a sus compañeros en las tres últimas dimensiones, y que el sexo del alumnado y la participación previa en actividades universitarias de servicio a la comunidad se configuran como variables que inciden en los niveles de desarrollo de estas competencias.

Palabras clave: aprendizaje-servicio; competencias cívicas; educación superior; alumnado universitario; educación inclusiva; profesionales de la educación

Resum. Aprenentatge servei $i$ desenvolupament civicosocial en titulacions universitàries de l'àmbit educatiu: preparant l'alumnat per practicar una educació inclusiva

En aquesta recerca s'hi analitza el potencial que té la metodologia de l'aprenentatge-servei $(\mathrm{ApS})$ per desenvolupar competències de caràcter civicosocial vinculades amb l'exercici d'una pràctica inclusiva en futurs professionals de l'educació. Seguint un disseny quasiexperimental (pretest-posttest), es compara els alumnes de titulacions de Ciències de l'Educació de la Universitat de Santiago de Compostela (USC) participants en experiències d'ApS amb aquells que segueixen una via formativa més convencional. La mostra està

* Este trabajo se deriva de dos proyectos del Programa Estatal de I+D+i Orientado a los Retos de la Sociedad (EDU2013-41687-R y EDU2017-82629-R) <http://www.usc.es/apsuni>. 
conformada per un total de 336 subjectes (188 en el grup experimental i 148 en el control). Específicament, s'hi analitzen les dimensions de lideratge, conducta prosocial, competència intercultural i treball en equip. Els resultats demostren que els qui participen en ApS acaben superant els seus companys en les tres últimes dimensions, i que el sexe de l'alumnat i la participació prèvia en activitats universitàries de servei a la comunitat es configuren com a variables que incideixen en els nivells de desenvolupament d'aquestes competències.

Paraules clau: aprenentatge-servei; competències cíviques; educació superior; alumnat universitari; educació inclusiva; professionals de l'educació

Abstract. Service-learning and civic and social development in education degrees: Preparing students for inclusive educational practice

This research analyzes the potential of service-learning (SL) methodology to develop civic and social competences related to the development of inclusive practice in future educational professionals. Using a quasi-experimental design (pretest-posttest), students in education degrees at the Santiago de Compostela University (USC), Spain, who participate in SL experiences are compared with those who follow a more conventional training path. The data-producing sample comprised 336 subjects (188 in the experimental group and 148 in the control one). Specifically, the dimensions of leadership, prosocial behavior, intercultural competence, and teamwork are analyzed. The results show that those who participate in SL surpass their peers in the last three competences. Additionally, the gender of the students and previous participation in university community service activities are variables that influence the development of these competences.

Keywords: service-learning; civic competences; higher education; university students; inclusive education; educational professionals

\begin{aligned} & \multicolumn{2}{c}{ Sumario } \\ & 1. Introducción 4. Resultados \\ & 2. El aprendizaje-servicio en las 5. Conclusiones \\ & titulaciones de educación: aprendizajes Referencias bibliográficas \\ & cívicos para una educación inclusiva \end{aligned}

3. Método

\section{Introducción}

Actualmente, la universidad está inmersa en un proceso de transformación que ha tenido claras consecuencias en el plano institucional y social, pero especialmente en el educativo. La creación del Espacio Europeo de Educación Superior (EEES) ha supuesto una modificación de las dinámicas universitarias, lo cual ha derivado en considerables cambios, sobre todo en términos de innovación educativa y fortalecimiento de la dimensión social del aprendizaje. La educación superior asume ahora la misión de formar al alumnado de un modo integral, atendiendo no solo a las competencias específicas referidas a su titu- 
lación, sino también a otras de carácter transversal enfocadas hacia el desarrollo personal y social (González y Wagenaar, 2003). Así se reconoce en las recientes declaraciones derivadas del EEES, caso de las surgidas en las reuniones de Bucarest (2012), Yerevan (2015) o París (2018), en las que se promueve la introducción de iniciativas de innovación educativa centradas en el aprendizaje del estudiante y el fortalecimiento de la dimensión social.

Consecuentemente, son numerosas las metodologías que comienzan a utilizarse con la intención de afrontar este nuevo paradigma formativo en el ámbito universitario, donde el aprendizaje se produce como resultado del esfuerzo y el trabajo autónomo de los estudiantes (Gargallo et al., 2011). Todo ello sin olvidar el potencial de las conexiones con el entorno comunitario, que contribuyen a fortalecer el desarrollo cívico-social del alumnado.

Una de las estrategias educativas que da respuesta a este modelo pedagógico y que con mayor fuerza ha calado en las instituciones universitarias españolas en los últimos años es el aprendizaje-servicio (ApS). Esta metodología supone la introducción de un servicio a la comunidad en las tradicionales tareas curriculares, incluyendo un componente de reflexión continua que permite establecer relaciones recíprocas entre el aprendizaje académico y la acción en entornos comunitarios. Precisamente por ello, su potencial reside en la adquisición de contenidos disciplinarios en complementación con el desarrollo cívico de los estudiantes, fruto de su participación en cuestiones de alcance social.

La capacidad para promover el enlace entre entidades sociales y educativas se ve especialmente acentuada en titulaciones de Ciencias de la Educación, donde esta metodología ha experimentado una rápida extensión. El ApS no solo difumina la fragmentación que solía caracterizar a estos grados universitarios en cuanto a formación en conocimientos y praxis social, sino que también favorece en el alumnado el compromiso con la profesión y promueve rasgos esenciales para la acción pedagógica, tales como la reciprocidad, la reflexión y la participación activa (Puig y Palos, 2006). Además, abre la puerta a la aparición, en el contexto académico, de actitudes cooperativas, de respeto mutuo y de solidaridad (Tejada, 2013).

Cabe destacar, también, que uno de sus objetivos es el desarrollo de competencias que permitirán a los futuros profesionales de la educación afrontar con éxito la creación de entornos de aprendizaje inclusivos (en ámbitos formales o no). Es ahí donde reside una de sus principales aportaciones, ya que, además de la adquisición de los conocimientos disciplinarios en los contextos en que se hacen operativos, permite a estos estudiantes experimentar situaciones reales en las que consolidar sus aprendizajes.

Así pues, el objetivo del presente trabajo es evaluar la eficacia del aprendizaje-servicio para mejorar las competencias cívico-sociales del alumnado en titulaciones universitarias de educación, bajo la premisa de que el desarrollo cívico garantizará una mayor predisposición a la aceptación de la diversidad que define a los contextos educativos formales y no formales, así como la potenciación de ambientes inclusivos. Para ello, se presentan los resultados de 
una investigación con estudiantes de los grados en Educación Infantil, Educación Primaria, Pedagogía y Educación Social, de la Universidad de Santiago de Compostela (USC), que participaron en proyectos de ApS. Concretamente, se analiza el impacto que tiene en variables mediadoras del desarrollo cívico, identificando, además, aquellos perfiles de estudiantes en los que el ApS demuestra mayor efectividad al respecto.

\section{El aprendizaje-servicio en las titulaciones de educación: aprendizajes cívicos para una educación inclusiva}

El aprendizaje-servicio se configura como una estrategia educativa que apunta no solo hacia dinámicas de desarrollo cognitivo y académico, sino también social y profesional. Una definición completa es la ofrecida por Naval et al. (2011):

Propuesta pedagógica que se dirige a la búsqueda de fórmulas concretas para implicar al alumnado en la vida cotidiana de las comunidades, barrios, instituciones cercanas. Se conceptualiza dentro de la educación experiencial y se caracteriza por: a) protagonismo del alumnado; b) atención a una necesidad real; c) conexión con objetivos curriculares; d) ejecución del proyecto de servicio y e) reflexión. (p. 88)

Es justo insistir aquí en el carácter curricular de la metodología, orientada a la adquisición de aprendizajes que serán evaluados y reconocidos en un marco académico, a diferencia de otras experiencias similares de educación no formal, como el voluntariado. Se trata, por tanto, de introducir en las rutinas de los diferentes niveles educativos iniciativas que impliquen al alumnado en la vida comunitaria. Así, se dota de significatividad a los aprendizajes de tipo disciplinario y técnico a través de la propia acción, a la vez que se promueve la adquisición de competencias de corte social.

De este modo, su adaptabilidad al nuevo modelo de universidad, donde se priorizan los procesos formativos centrados en la autonomía de los estudiantes, parece incuestionable. Y es que, entre otros, facilita la puesta en práctica de los saberes teóricos adquiridos en el aula, sitúa los conocimientos disciplinarios en los entornos en los que resultan funcionales y útiles, familiariza a los jóvenes universitarios con las exigencias del mundo del trabajo y las organizaciones, y fortalece la formación en valores (Tejada, 2013).

En nuestro país, la propia CRUE apostó por la inclusión del ApS en las aulas universitarias, para lo cual realizó un llamamiento a la promoción de procesos rigurosos de institucionalización de la metodología. Su propuesta se ampara en la capacidad para impulsar políticas de responsabilidad social en las instituciones, así como para trabajar las competencias de corte cívico-social en la educación superior (CADEP-CRUE, 2015). No en vano, han sido numerosas las universidades que han emprendido dinámicas de institucionalización sistemáticas y continuadas (Naval y Arbués, 2018; Santos Rego y Lorenzo, 2018) desde un paradigma que enfatiza la necesidad de una práctica concienciada e inclusiva. 
Pero el aprendizaje-servicio no ha tenido la misma aceptación en todas las disciplinas y, a pesar de su extensión en la práctica totalidad de titulaciones universitarias, parece que ha sido en las ciencias sociales, más aún en la rama de educación, donde mayor expansión ha experimentado. En concreto, en estas titulaciones se ha enfatizado su componente axiológico, pues sitúa al alumnado en contacto con diferentes necesidades y problemáticas enraizadas en su comunidad más cercana (Aramburuzabala y García, 2012). Al mismo tiempo, permite conformar un conjunto de principios éticos y deontológicos que resultan ser de gran trascendencia para los profesionales del ámbito educativo, en torno a los cuales ha de articularse el auténtico ejercicio de una educación inclusiva.

Actualmente, la escuela inclusiva es todavía un reto de la práctica pedagógica. Este paradigma supone el reconocimiento del valor de la diversidad que caracteriza a cada ser humano (Peñalva y Leiva, 2019), para lo cual es imprescindible atender a cada alumno de forma individualizada - que no individual- . Se trata, por tanto, de un proceso en permanente adaptación (Durán y Giné, 2011), en el que la capacidad de respuesta de los centros educativos tiene un peso fundamental en el reconocimiento de las singularidades propias de cada estudiante, puesto que pueden ofrecer más y mejores oportunidades para todo el alumnado. En este contexto, la formación del conjunto de los profesionales de la educación resulta esencial, ya que se constituyen como los artífices de esos nuevos espacios de aprendizaje: ellos son los facilitadores de la construcción de una educación en la que calidad e inclusión son variables recíprocas (Muntaner, Rosselló y De la Iglesia, 2016).

Además, la educación inclusiva puede ser vista desde una perspectiva más amplia, en la que se mire más allá de los muros de la escuela. Estamos hablando de la Educación para Todos, iniciativa propuesta por la UNESCO y que apunta a un compromiso global de educación de calidad para todas las personas de cualquier edad y condición. Justamente, este es el cuarto de los Objetivos de Desarrollo Sostenible 2030, donde la alfabetización universal, la educación para la ciudadanía y la construcción de entornos de aprendizaje eficaces comparten un mismo fin: la inclusión social.

Empero, pocas dudas quedan de que este paradigma requiera de cambios a nivel metodológico y organizativo, puesto que su implementación depende de unos profesionales sensibilizados ante la creación, a través de la educación, de una sociedad más equitativa, justa y respetuosa; motivo más que suficiente para justificar que las titulaciones universitarias de educación asuman nuevas metodologías y dinámicas formativas que lleven el conocimiento técnico un paso más allá, incidiendo también en el desarrollo cívico-social y axiológico de los estudiantes (Santos Rego, Sotelino y Lorenzo, 2015). En este sentido, nuestro trabajo trata de evidenciar que, a través del aprendizaje-servicio y desde un paradigma inclusivo, es posible construir un marco potencialmente significativo para que el alumnado desarrolle competencias que luego podrá desplegar en el ámbito profesional para conseguir una sociedad más justa y equitativa. 
Este enfoque metodológico permite proponer el aprendizaje cívico como un objetivo de primer nivel, a diferencia de otras metodologías para las que el desarrollo académico y cognitivo suele ser la finalidad principal (Bringle y Clayton, 2012). Consecuentemente, numerosas investigaciones han constatado su eficacia en la adquisición de competencias cívico-sociales, entendidas estas como «un conglomerado de factores cognitivos y socio-afectivos (incluyendo conductas, destrezas comunicativas, habilidades interactivas, etc.), que propulsan una disposición individual a ser, estar y sentirse de una manera determinada, pero siempre susceptible de ser entendida según patrones de vida comunitaria» (Santos Rego y Lorenzo, 2010, p. 10).

Lo que estos estudios vienen a constatar es la incidencia del aprendizajeservicio en dimensiones como la acción cívica, las competencias interpersonales y de resolución de problemas, la conciencia política, el liderazgo, las actitudes de justicia social o la competencia intercultural (Einfeld y Collins, 2008; Moely et al., 2002), sin olvidar el impacto en los niveles de participación social de los estudiantes (Sotelino, Mella y Rodríguez, 2019).

Centrándonos ya en las titulaciones de Ciencias de la Educación, Bernadowski, Perry y Del Greco (2013) afirman que el aprendizaje-servicio permite a los futuros maestros desarrollarse como profesionales reflexivos, competentes en la resolución de problemas y copartícipes en las relaciones de los centros escolares con su entorno comunitario. En otras palabras, este tipo de experiencias dan lugar a situaciones que incitan a los estudiantes a reflexionar críticamente sobre los diferentes contextos sociales, reconociendo las dinámicas de inclusión y exclusión que se producen en ellos (Martínez y Martínez, 2015).

La investigación de Carrington y Saggers (2008), por su parte, apunta a una mejora significativa en el respeto hacia la diversidad y la deconstrucción de prejuicios en maestros en formación que participan en iniciativas de aprendizaje-servicio. Concretamente, los estudiantes conforman un marco ético en torno al cual podrán ejercer procesos educativos inclusivos.

Del mismo modo, también con estudiantes de educación, Root, Callahan y Sepanski (2002) constatan un incremento en las actitudes de aceptación de la diversidad, así como una mayor predisposición ética a servir a la comunidad. Por el mismo sendero discurre la investigación de Carrington et al. (2015), en el que un grupo de maestros en formación afirma que la participación en ApS incide directamente en su futura práctica docente, pues los familiariza con una enseñanza inclusiva y fomenta valores, como el respeto y la empatía, que inciden en la aceptación de la diversidad.

En España, numerosos estudios han demostrado el potencial de esta metodología en las diversas titulaciones de Ciencias de la Educación. Por ejemplo, Gil, Chiva y Martí (2013) evidencian que el contacto directo con la comunidad permite a alumnos del grado en Maestro desarrollar los principios en los que se vertebra la escuela inclusiva, en tanto que adquieren un compromiso ético y moral con todos los destinatarios del servicio. También en la formación inicial docente destaca la obra coordinada por Chiva-Bartoll y Gil-Gómez 
(2018), donde se recopilan experiencias en las que se utilizó el aprendizajeservicio en diferentes universidades españolas.

Por otro lado, en el grado en Educación Social, Martínez y Martínez (2015) describen cómo una experiencia que se centra en la brecha digital fomenta el desarrollo cívico y personal de los participantes, enfatizando el papel que juega su profesión a la hora de mitigar la exclusión social de colectivos en riesgo. Finalmente, el proyecto del que nos informan Sotelino, Santos Rego y García Álvarez (2019), en el que alumnos de Pedagogía trabajan en aras de favorecer la inclusión social de la población inmigrante, contribuye a desarrollar un conjunto de competencias cívicas cuya aportación al futuro ejercicio profesional es altamente valorada por los estudiantes.

Con mayor nivel de concreción, en el contexto español también se ha prestado atención a las posibilidades que emanan de la utilización del aprendizaje-servicio en las titulaciones universitarias del ámbito educativo a fin de trabajar la educación inclusiva, siendo buen ejemplo de ello los estudios de Vázquez, Liesa y Lozano (2017) y Ruiz-Bejarano (2020). De esta forma, más allá de su indudable potencial profesionalizante en la formación del alumnado de Ciencias de la Educación, el ApS se articula como una metodología privilegiada para fomentar el desarrollo de aprendizajes cívicos, todo ello a través de un contacto directo con la propia comunidad. Estas competencias, totalmente necesarias para la futura práctica pedagógica, se erigen como la única vía para alcanzar la transformación de la sociedad a través de un paradigma inclusivo, finalidad última de la educación y, por tanto, de su futuro ejercicio profesional.

\section{Método}

La investigación que aquí se presenta pretende conocer la existencia de diferencias en los niveles de desarrollo de competencias de tipo cívico-social en el alumnado de Ciencias de la Educación, en función de su implicación en proyectos de aprendizaje-servicio. Para ello, se utilizó un diseño cuasiexperimental, donde la participación en estos proyectos se conformó como la variable independiente, aplicando un cuestionario de pretest y postest a dos grupos: el experimental (GE), conformado por los estudiantes que se involucraron en el aprendizaje-servicio, y el control (GC), que incluía a aquellos que no lo hicieron.

Para acceder a los participantes, se ha optado por un muestreo bietápico. En primer lugar, a través de un muestreo intencionado no probabilístico, se seleccionaron las dos facultades en las que se imparten las titulaciones del ámbito educativo en la USC: Ciencias de la Educación y Formación del Profesorado. A continuación, se procedió de nuevo con el mismo tipo de muestreo, pues la selección de los estudiantes participantes en aprendizaje-servicio y de sus compañeros de curso dependió directamente de los proyectos concretos que se estaban desarrollando en estas titulaciones. Finalmente, se analizaron las asignaturas donde se enmarcaban las siete experiencias que se implemen- 
taron durante el curso 2016-2017. En total, se contó con 478 respuestas en el pretest del cuestionario y con 416 en el postest. Sin embargo, la muestra final se redujo a 336 sujetos, que son los que coinciden en ambas aplicaciones.

De este total de 336 participantes, 188 (un 56\%) se implicaron en proyectos de aprendizaje-servicio (grupo experimental), mientras que los 148 restantes (un 44\%) siguieron la metodología convencional (grupo control). Las edades se sitúan entre los 18 y los 41 años, si bien el 78,9\% se encuentra en el tramo $18-22$, con una media de 21,05 años. Se halla una presencia predominante de mujeres, lo que da cuenta de la feminización de las titulaciones del ámbito educativo: 276 mujeres (un 82,1\%) frente a 60 hombres (un 17,9\%).

La mayor parte de los alumnos procede del grado en Maestro de Educación Primaria (un 67,6\%) y los demás se distribuyen equitativamente entre los grados en Maestro de Educación Infantil (un 10,1\%), Educación Social (un $10,7 \%$ ) y Pedagogía (un 11,6\%). Asimismo, también se da un reparto equilibrado de la muestra a lo largo de los cuatro cursos de las titulaciones: un $19,9 \%$ cursan primero; un $26,2 \%$, segundo; un $32,4 \%$, tercero, y un $21,4 \%$, cuarto. Por último, es importante destacar que 38 sujetos (un 11,3\%) han participado anteriormente en proyectos promovidos desde la Universidad que implicaban algún tipo de servicio a la comunidad.

El instrumento utilizado es el Cuestionario sobre Competencias CívicoSociales y Autoeficacia del Alumnado Universitario (CUCOCSA) (Santos Rego y Lorenzo, 2018), previamente validado (Mella, 2019). En concreto, se empleó la escala referida a las competencias cívico-sociales, formada por un total de 20 ítems que, tras la extracción inicial derivada del análisis factorial exploratorio, se organizaron en cuatro factores significativos que explican una varianza del 50,96\%: conducta prosocial $(\alpha=0,79)$, liderazgo $(\alpha=0,72)$, competencia intercultural $(\alpha=0,73)$ y trabajo en equipo y relación con los demás $(\alpha=0,70)$. A continuación, se realizó un análisis factorial confirmatorio con el que se comprobó que la estructura factorial obtenida representaba adecuadamente la información proporcionada por los datos (Santos Rego et al., 2020).

\section{Resultados}

Comenzaremos el análisis reparando en las diferencias que se presentan entre las medias de los estudiantes en las dos aplicaciones del instrumento, a fin de conocer el modo en que evolucionaron durante el período evaluado. Los datos indican que, en los cuatro factores que conforman la escala empleada, ambos grupos parten de puntuaciones ya muy elevadas. La excepción es la dimensión de liderazgo, en la que todos los alumnos (GE y GC) experimentan cambios significativos entre la aplicación del pretest y la del postest (tablas 1 y 2).

Mientras que «Liderazgo» es el único factor de la escala en el que los alumnos que no participan en los proyectos de ApS experimentan un avance significativo, aquellos que sí se implicaron en estas experiencias también incrementan los resultados obtenidos en "Trabajo en equipo y relación con los demás» 
Tabla 1. Diferencias entre el pretest y el postest del grupo experimental

\begin{tabular}{|c|c|c|c|c|c|c|c|}
\hline \multirow{3}{*}{ Factor } & \multicolumn{7}{|c|}{ Grupo experimental } \\
\hline & \multicolumn{2}{|c|}{ Pretest } & \multicolumn{2}{|c|}{ Postest } & \multirow{2}{*}{$t$} & \multirow{2}{*}{ gl } & \multirow{2}{*}{$p$} \\
\hline & Media & $D T$ & Media & DT & & & \\
\hline Conducta prosocial & 4,51 & 0,401 & 4,53 & 0,406 & $-0,850$ & 182 & 0,397 \\
\hline Liderazgo & 3,80 & 0,533 & 3,90 & 0,515 & $-2,679$ & 183 & 0,008 \\
\hline Competencia intercultural & 4,32 & 0,576 & 4,39 & 0,597 & $-1,597$ & 184 & 0,112 \\
\hline $\begin{array}{l}\text { Trabajo en equipo y relación con } \\
\text { los demás }\end{array}$ & 4,32 & 0,483 & 4,40 & 0,503 & $-2,149$ & 186 & 0,033 \\
\hline
\end{tabular}

Fuente: elaboración propia.

Tabla 2. Diferencias entre el pretest y el postest del grupo control

\begin{tabular}{|c|c|c|c|c|c|c|c|}
\hline \multirow{3}{*}{ Factor } & \multicolumn{7}{|c|}{ Grupo control } \\
\hline & \multicolumn{2}{|c|}{ Pretest } & \multicolumn{2}{|c|}{ Postest } & \multirow{2}{*}{$t$} & \multirow{2}{*}{$g l$} & \multirow{2}{*}{$p$} \\
\hline & Media & $D T$ & Media & $D T$ & & & \\
\hline Conducta prosocial & 4,42 & 0,373 & 4,46 & 0,371 & $-1,238$ & 144 & 0,218 \\
\hline Liderazgo & 3,59 & 0,603 & 3,76 & 0,553 & $-4,080$ & 144 & 0,000 \\
\hline Competencia intercultural & 4,23 & 0,552 & 4,23 & 0,568 & 0,000 & 146 & 1,000 \\
\hline $\begin{array}{l}\text { Trabajo en equipo y relación con } \\
\text { los demás }\end{array}$ & 4,25 & 0,531 & 4,27 & 0,544 & $-0,626$ & 144 & 0,532 \\
\hline
\end{tabular}

Fuente: elaboración propia.

(tabla 1). Se presenta a continuación la figura 1, a fin de facilitar la comprensión de los resultados obtenidos por ambos grupos en las dos aplicaciones del cuestionario.

Sin embargo, los resultados más destacados se manifiestan al término de los proyectos (postest), pues los datos muestran diferencias a favor del grupo de ApS en tres de los cuatro factores estudiados. Ahora bien, la prudencia obliga a analizar dichos resultados tomando en consideración, además, la situación que demostraban en la aplicación del pretest (tablas 3 y 4). En concreto, de los tres factores en los que el alumnado implicado en ApS termina superando a sus compañeros («Liderazgo», "Competencia intercultural», $y$ «Trabajo en equipo y relación con los demás»), solamente podemos atribuir a la participación en estos proyectos los dos últimos, pues en el caso del liderazgo las diferencias entre grupos ya existían en la aplicación del pretest. 
Figura 1. Puntuaciones obtenidas por el GE y el GC en el pretest y el postest

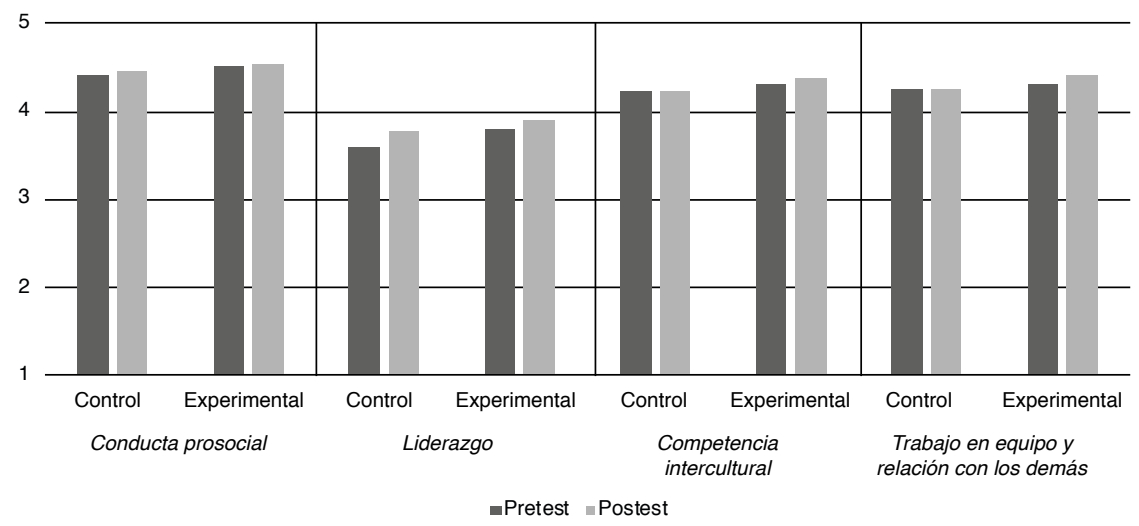

Fuente: elaboración propia.

Tabla 3. Diferencias entre el GE y el GC en el pretest

\begin{tabular}{|c|c|c|c|c|c|}
\hline \multirow{2}{*}{ Factor } & \multicolumn{2}{|c|}{$\begin{array}{l}\text { Prueba de Levene de } \\
\text { igualdad de varianzas }\end{array}$} & \multirow[t]{2}{*}{$t$} & \multirow{2}{*}{$g l$} & \multirow{2}{*}{$\begin{array}{c}\text { Sig. } \\
\text { (bilateral) }\end{array}$} \\
\hline & $\mathbf{F}$ & Sig. & & & \\
\hline Conducta prosocial & 0,285 & 0,594 & $-2,283$ & 332 & 0,023 \\
\hline Liderazgo & 2,018 & 0,156 & $-3,298$ & 331 & 0,001 \\
\hline Competencia intercultural & 1,428 & 0,233 & $-1,366$ & 332 & 0,173 \\
\hline $\begin{array}{l}\text { Trabajo en equipo y relación con } \\
\text { los demás }\end{array}$ & 0,140 & 0,709 & $-1,281$ & 331 & 0,201 \\
\hline
\end{tabular}

Fuente: elaboración propia.

Tabla 4. Diferencias entre el GE y el GC en el postest

\begin{tabular}{|c|c|c|c|c|c|}
\hline \multirow{2}{*}{ Factor } & \multicolumn{2}{|c|}{$\begin{array}{l}\text { Prueba de Levene de } \\
\text { igualdad de varianzas }\end{array}$} & \multirow{2}{*}{$t$} & \multirow{2}{*}{$g l$} & \multirow{2}{*}{$\begin{array}{c}\text { Sig. } \\
\text { (bilateral) }\end{array}$} \\
\hline & $\mathbf{F}$ & Sig. & & & \\
\hline Conducta prosocial & 0,554 & 0,457 & $-1,519$ & 328 & 0,130 \\
\hline Liderazgo & 0,451 & 0,502 & $-2,338$ & 330 & 0,020 \\
\hline Competencia intercultural & 0,637 & 0,425 & $-2,425$ & 332 & 0,016 \\
\hline $\begin{array}{l}\text { Trabajo en equipo y relación con } \\
\text { los demás }\end{array}$ & 1,038 & 0,309 & $-2,075$ & 333 & 0,039 \\
\hline
\end{tabular}

Fuente: elaboración propia. 
A continuación se examinó el perfil de estudiante en el que el ApS había comportado un efecto mayor. Para ello, se estudiaron las diferencias que se daban dentro de las puntuaciones del postest del GE en función de las siguientes variables: sexo; ciclo; carácter obligatorio o voluntario de los diferentes proyectos; participación previa de los estudiantes en algún tipo de iniciativa, promovida desde la universidad, que supusiese un servicio a la comunidad, y titulación. Así, aparecen diferencias en función del sexo del alumnado y de la participación previa en otras actividades universitarias de servicio a la comunidad.

En concreto, en el primer caso, las mujeres demuestran puntuaciones superiores, tanto en conducta prosocial $\left(\overline{\mathrm{x}}_{\text {Mujeres }}-\overline{\mathrm{x}}_{\text {Hombres }}=0,24, p=0,002\right)$ como en competencia intercultural $\left(\overline{\mathrm{x}}_{\text {Mujeres }}-\overline{\mathrm{x}}_{\text {Hombres }}=0,31, p=0,006\right)$. Por otra parte, quienes habían participado previamente en actividades universitarias de servicio a la comunidad cuentan con mayores niveles de conducta prosocial $\left(\overline{\mathrm{x}}_{\text {SíParticipación }}-\overline{\mathrm{x}}_{\text {NoParticipación }}=0,26, p=0,000\right)$.

\section{Conclusiones}

El eje de nuestro estudio ha sido conocer la eficacia del aprendizaje-servicio para promover mejoras en el nivel de desarrollo de las competencias cívicosociales del alumnado de Ciencias de la Educación. Al pivotar todas ellas en torno a dimensiones como la conducta prosocial; el liderazgo; la competencia intercultural, y el trabajo en equipo y la relación con los demás, consideramos que facilitarán, en el caso de los profesionales del ámbito educativo, una mayor predisposición a asumir en la práctica los principios básicos de la atención a la diversidad en el marco de una educación inclusiva (Peñalva y Leiva, 2019).

Los resultados presentados permiten constatar que los estudiantes que participan en este tipo de proyectos terminan manifestando puntuaciones significativamente más altas que los compañeros que siguen las vías formativas convencionales en la mayoría de las competencias cívico-sociales evaluadas, lo que confirma lo demostrado por estudios como el de Moely et al. (2002) y Einfeld y Collins (2008).

En concreto, destacan las diferencias que se dan en el postest en referencia a la competencia intercultural y al trabajo en equipo y la relación con los demás, pues las puntuaciones en la medida del pretest corrían parejas en ambos grupos. En cuanto a la competencia intercultural, este trabajo confirma la efectividad del aprendizaje-servicio para desarrollar la capacidad intercultural del alumnado en términos de competencia (conocimientos, procedimientos y actitudes) (Holsapple, 2012). Por lo que respecta al trabajo en equipo y la relación con los demás, concordamos con las mejoras que se demostraron en el estudio de García García y Sánchez Calleja (2017) en cuanto a competencia social.

También hemos de destacar que en nuestro estudio se localizaron diferencias entre los estudiantes del grupo de $\mathrm{ApS}$ y sus compañeros con respecto al liderazgo, aunque no podemos asociarlas a la implicación en este tipo de pro- 
yectos. Esta dimensión, siguiendo a Moely e Ilustre (2014), solo puede ser desarrollada adecuadamente cuando los proyectos no se limitan a actos caritativos, sino que apuntan hacia una auténtica transformación social.

Asimismo, se han observado diferencias entre los propios participantes en experiencias de ApS, concretamente en función del sexo (favorables a las mujeres) y en la participación en actividades universitarias de servicio a la comunidad (favorables a quienes se habían implicado en este tipo de iniciativas). Los mejores resultados a favor de las mujeres en la dimensión social también han sido constatados en el trabajo de Moely e Ilustre (2019), mientras que Brandes y Randall (2011) confirmaron que los alumnos que habían participado previamente en esta estrategia educativa estaban predispuestos a obtener mejores resultados en cuestiones de tipo cívico.

Es aquí donde queremos justificar la pertinencia del aprendizaje-servicio como una herramienta en la que el desarrollo cognitivo se ve complementado con una proyección cívica y social del aprendizaje. En la sociedad actual, y a la luz de las recomendaciones que llegan desde el proceso de Bolonia, la universidad ha de contribuir directamente a promover el desarrollo personal y profesional de los estudiantes, así como a construir un marco ético y axiológico acorde a las demandas de la educación inclusiva, reconociendo la insuficiencia de un aprendizaje excesivamente técnico y disciplinario alejado de la práctica comunitaria. Precisamente por ello urgen metodologías que, como el aprendizaje-servicio, permitan afrontar la educación universitaria no solo desde una perspectiva más integral, que garanticen mejores condiciones para los egresados en el mundo laboral (Santos Rego et al., 2015), sino también desde su finalidad transformadora, lo cual dotará al alumnado de las competencias necesarias para el ejercicio de una práctica inclusiva en contextos de diversidad (Carrington y Saggers, 2008; Root et al., 2002).

El desarrollo cívico y social de los estudiantes universitarios es una cuestión que debe importar a todas las disciplinas, independientemente del área de conocimiento en la que se sitúen. En el caso de nuestro estudio enfatizamos la importancia que tienen estas competencias para el alumnado de titulaciones de educación, donde los conocimientos, las habilidades y las actitudes con orientación social y cívica han de marcar la diferencia en su seña de identidad profesional. Incluso, podría decirse que este colectivo es el protagonista del proceso de construcción de una educación y una sociedad más inclusivas, justas e igualitarias (Muntaner et al., 2016).

En este sentido, el contacto con la comunidad dispone de situaciones en las que los futuros profesionales de la educación podrán reconocer dinámicas sociales más amplias, más allá de los principios abstractos y teóricos que se trabajan en el aula, y asumir como propia la función de intervenir sobre las desigualdades que pueden generarse en el seno de las instituciones formativas. Es más, cabe preguntarse aquí sobre qué tipo de principios inclusivos pueden llegar a aprenderse si no es a través de la propia experiencia, con metodologías que ubiquen al alumnado en situaciones sobre las que reflexionar de forma crítica y, consecuentemente, desarrollar aprendizajes significativos. A la luz de 
los resultados obtenidos, el aprendizaje-servicio se erige como una práctica que permite trabajar en esta línea, en tanto que fomenta una serie de competencias cívico-sociales clave para su práctica profesional.

Finalmente, la última aportación de nuestro estudio radica en su contribución al avance institucional del aprendizaje-servicio en la universidad española. Por tanto, lo que realmente importa, más allá de discursos teóricos que giran en torno al potencial que la metodología ha evidenciado en otros países, es la demostración de sus bondades con respaldo empírico, para lo cual han de desarrollarse diseños rigurosos y evaluar su puesta en práctica. Se trata, consecuentemente, de justificar con estudios sistemáticos las virtudes del ApS en diferentes dimensiones del aprendizaje universitario: cognitivo y académico (en tanto que se erige como una metodología de trabajo curricular); ético y social (al disponer de situaciones en las que se interactúa con la comunidad para hacer frente a las necesidades que en ella radican), y profesional (pues sitúa al alumnado en contacto con realidades próximas a su campo de trabajo). Solamente así se podrá garantizar un adecuado desarrollo de la metodología, especialmente en lo que se refiere a los niveles de calidad de las experiencias.

\section{Referencias bibliográficas}

Aramburuzabala, P. y García, R. (2012). El aprendizaje-servicio en la formación de maestros. Revista del Congrés Internacional de Docència Universitària i Innovació, 1, 1-15. Recuperado de <https://www.cidui.org/revistacidui/index.php/cidui/ article/download/232/221.pdfs.

Bernadowski, C.; Perry, R. y Greco, R. del (2013). Improving preservice teachers' self-efficacy through service learning: Lessons learned. International Journal of Instruction, 6(2), 67-86.

Brandes, K. y Randall, G.K. (2011). Service learning and civic responsibility: Assessing aggregate and individual level change. International Journal of Teaching and Learning in Higher Education, 23(1), 20-29.

Bringle, R.G. y Clayton, P.H. (2012). Civic education through service learning: What, how, and why? En L. McIlrath, A. Lyons y R. Munck (eds.). Higher education and civic engagement: Comparative perspectives (pp. 101-124). Nueva York, NY: Palgrave Macmillan.

CADEP-CRUE (2015). Institucionalización del Aprendizaje-Servicio como estrategia docente dentro del marco de la Responsabilidad Social Universitaria para la promoción de la Sostenibilidad en la Universidad. Recuperado de <https://www.crue.org/ wp-content/uploads/2020/02/2.-APROBADA-INSTITUCIONALIZACIONApS.pdf>

Carrington, S.; Mercer, K.L.; Iyer, R. y Selva, G. (2015). The impact of transformative learning in a critical service-learning program on teacher development: Building a foundation for inclusive teaching. Reflective Practice, 16(1), 61-72. <https://doi.org/10.1080/14623943.2014.969696>

Carrington, S. y Saggers, B. (2008). Service-learning informing the development of an inclusive ethical framework for beginning teachers. Teaching and Teacher Education, 24(3), 795-806. $<$ https://doi.org/10.1016/j.tate.2007.09.006> 
Chiva-Bartoll, O. y Gil-Gómez, J. (eds.) (2018). Aprendizaje-servicio universitario: Modelos de intervención e investigación en la formación inicial docente. Barcelona: Octaedro.

Durán, D. y Giné, C. (2011). La formación del profesorado para la educación inclusiva: Un proceso de desarrollo profesional y de mejora de los centros para atender la diversidad. Revista Latinoamericana de Educación Inclusiva, 5(2), 153-170.

Einfeld, A. y Collins, D. (2008). The relationships between service-learning, social justice, multicultural competence, and civic engagement. Journal of College Student Development, 49(2), 95-109. <https://doi.org/10.1353/csd.2008.0017>

García García, M. y Sánchez Calleja, L. (2017). El aprendizaje servicio y el desarrollo de las competencias emocionales en la formación inicial del profesorado. Contextos Educativos, 20, 127-145. <http://doi.org/10.18172/con.2991>

Gargallo, B.; Suárez, J.; Garfella, P.R. y Fernández March, A. (2011). El cuestionario CEMEDEPU: Un instrumento para la evaluación de la metodología docente y evaluativa de los profesores universitarios. Estudios sobre Educación, 21, 9-40.

Gil, J.; Chiva, Ó. y Martí, M. (2013). La adquisición de la competencia social y ciudadana en la universidad mediante el aprendizaje-servicio: Un estudio cuantitativo y cualitativo en el ámbito de la educación física. Revista Internacional de Educación para la Justicia Social, 2(2), 89-108.

González, J. y Wagenaar, R. (2003). Tuning Educational Structures in Europe. Bilbao: Universidad de Deusto.

Holsapple, M.A. (2012). Service-learning and student diversity outcomes: Existing evidence and directions for future research. Michigan Journal of Community Service Learning, 18(2), 5-18.

Martínez, B. y Martínez, I. (2015). El aprendizaje servicio y la formación inicial de los profesionales de la educación. Profesorado: Revista de Currículum y Formación del Profesorado, 19(1), 244-260.

Mella, I. (2019). Aprendizaje-servicio y rendimiento académico del alumnado universitario: La evaluación de un programa. Universidad de Santiago de Compostela. Tesis doctoral. Recuperado de <https://minerva.usc.es/xmlui/handle/10347/ 20158>.

Moely, B.E. e Ilustre, V. (2014). The impact of service-learning course characteristics on university students' learning outcomes. Michigan Journal of Community Service Learning, 21(1), 5-16.

- (2019). Service involvement and civic attitudes of university alumni: Later-correlates of required public service participation during college. Michigan Journal of Community Service Learning, 25(1), 30-42. <https://doi.org/10.3998/mjcsloa.3239521.0025.103>

Moely, B.E.; Mercer, S.H.; Ilustre, V.; Miron, D. y MacFarland, M. (2002). Psychometric properties and correlates of the Civic Attitudes and Skills Questionnaire (CASQ): A measure of students' attitudes related to service-learning. Michigan Journal of Community Service Learning, 8(2), 15-26.

Muntaner, J.; Rosselló, R. e Iglesia, B. de la (2016). Buenas prácticas en educación inclusiva. Educatio Siglo XXI, 34(1), 31-50.

Naval, C. y Arbués, E. (eds.) (2018). Hacer la universidad en el espacio social. Pamplona: EUNSA. 
Naval, C.; García, R.; Puig, J. y Santos Rego, M.A. (2011). La formación éticocívica y el compromiso social de los estudiantes universitarios. Encounters on Education, 12, 77-91.

Peñalva, A. y Leiva, J.L. (2019). La interculturalidad en el contexto universitario: Necesidades en la formación inicial de los futuros profesionales de la educación. Educar, 55(1), 141-158. <https://doi.org/10.5565/rev/educar.989>

Puig, J.M. y PAlos, J. (2006). Rasgos pedagógicos del aprendizaje-servicio. Cuadernos de Pedagogía, 357, 60-63.

Root, S.; Callahan, J. y Sepanski, J. (2002). Building teaching dispositions and service-learning practice: A multi-site study. Michigan Journal of Community Service Learning, 8(2), 50-60.

Ruiz-Bejarano, A.M. (2020). Educación inclusiva, criticidad y compromiso social: Innovación docente y Aprendizaje-Servicio en la formación inicial docente. REDU: Revista de Docencia Universitaria, 18(1), 233-248. <https://doi.org/10.4995/redu.2020.12407>

Santos Rego, M.A.; Ferraces, M.J.; Mella, I. y Vázquez Rodríguez, A. (2020). Universidad, competencias cívico-sociales y mercado de trabajo. Revista Española de Pedagogía, 78(276), 213-232.

<https://doi.org/10.22550/REP78-2-2020-06>

Santos Rego, M.A. y Lorenzo, M. (2010). Dimensión cívica y desarrollo formativo de los estudiantes universitarios en el contexto español. Revista Electrónica de Investigación Educativa, número especial, 1-17.

Santos Rego, M.A. y Lorenzo, M. (eds.) (2018). Guía para la institucionalización del aprendizaje-servicio en la universidad. Santiago de Compostela: Servizo de Publicacións e Intercambio Científico da Universidade de Santiago de Compostela.

Santos Rego, M.A.; Sotelino, A. y Lorenzo, M. (2015). Aprendizaje-servicio y misión cívica de la universidad: Una propuesta de desarrollo. Barcelona: Octaedro.

Sotelino, A.; Mella, I. y Rodríguez, M.A. (2019). El papel de las entidades cívicosociales en el aprendizaje-servicio: Sistematizando la participación del alumnado en el tercer sector. Teoría de la Educación, 31(2), 197-219. <https://doi.org/10.14201/teri.20156>

Sotelino, A.; Santos Rego, M.A. y García Álvarez, J. (2019). El aprendizajeservicio como vía para el desarrollo de competencias interculturales en la Universidad. Educatio Siglo XXI, 37(1), 73-90. <https://doi.org/10.6018/educatio.363391>

Tejada, J. (2013). La formación de las competencias profesionales a través del aprendizaje-servicio. Cultura y Educación, 25(3), 285-294. <https://doi.org/10.1174/113564013807749669>

Vázquez, S.; Liesa, M. y Lozano, A. (2017). Recreos cooperativos e inclusivos a través de la metodología de Aprendizaje-Servicio. Revista Electrónica Interuniversitaria de Formación del Profesorado, 20(1), 173-185.

<https://doi.org/10.6018/reifop/20.1.213181> 